University of South Carolina

Scholar Commons

7-19-2011

\title{
Efficient Quantum Trajectory Representation of Wavefunctions Evolving in Imaginary Time
}

\author{
Sophya V. Garashchuk \\ University of South Carolina - Columbia, garashch@mailbox.sc.edu \\ James Mazzuca \\ University of South Carolina - Columbia, mazzuca@mailbox.sc.edu \\ Tijo Vazhappilly \\ University of South Carolina - Columbia
}

Follow this and additional works at: https://scholarcommons.sc.edu/chem_facpub

Part of the Chemistry Commons

\section{Publication Info \\ Published in The Journal of Chemical Physics, Volume 135, Issue 3, 2011, pages 034104-. \\ (c) The Journal of Chemical Physics 2011, American Institute of Physics.}

This Article is brought to you by the Chemistry and Biochemistry, Department of at Scholar Commons. It has been accepted for inclusion in Faculty Publications by an authorized administrator of Scholar Commons. For more information, please contact digres@mailbox.sc.edu. 


\section{University of South Carolina}

Scholar Commons

Faculty Publications

Chemistry and Biochemistry, Department of

2011

\section{Efficient quantum trajectory representation of wavefunctions evolving in imaginary time}

Sophya V. Garashchuk

James Mazzuca

Tijo Vazhappilly

Follow this and additional works at: http://scholarcommons.sc.edu/chem_facpub Part of the Chemistry Commons

This Article is brought to you for free and open access by the Chemistry and Biochemistry, Department of at Scholar Commons. It has been accepted for inclusion in Faculty Publications by an authorized administrator of Scholar Commons. For more information, please contact SCHOLARC@mailbox.sc.edu. 


\title{
Efficient quantum trajectory representation of wavefunctions evolving in imaginary time
}

\author{
Sophya Garashchuk, ${ }^{\text {a) }}$ James Mazzuca, and Tijo Vazhappilly \\ Department of Chemistry and Biochemistry, University of South Carolina, Columbia, \\ South Carolina 29208, USA
}

(Received 14 February 2011; accepted 22 June 2011; published online 19 July 2011)

\begin{abstract}
The Boltzmann evolution of a wavefunction can be recast as imaginary-time dynamics of the quantum trajectory ensemble. The quantum effects arise from the momentum-dependent quantum potential - computed approximately to be practical in high-dimensional systems - influencing the trajectories in addition to the external classical potential [S. Garashchuk, J. Chem. Phys. 132, 014112 (2010)]. For a nodeless wavefunction represented as $\psi(x, t)=\exp (-S(x, t) / \hbar)$ with the trajectory momenta defined by $\nabla S(x, t)$, analysis of the Lagrangian and Eulerian evolution shows that for bound potentials the former is more accurate while the latter is more practical because the Lagrangian quantum trajectories diverge with time. Introduction of stationary and time-dependent components into the wavefunction representation generates new Lagrangian-type dynamics where the trajectory spreading is controlled improving efficiency of the trajectory description. As an illustration, different types of dynamics are used to compute zero-point energy of a strongly anharmonic well and low-lying eigenstates of a high-dimensional coupled harmonic system. () 2011 American Institute of Physics. [doi:10.1063/1.3610165]
\end{abstract}

\section{INTRODUCTION}

Some recent research, focused on the quantum or the Madelung-de Broglie-Bohm trajectory formulation of the time-dependent Schrödinger equation, ${ }^{1-3}$

$$
\hat{H} \psi(x, t)=\imath \hbar \frac{\partial}{\partial t} \psi(x, t),
$$

as an alternative to conventional "exact" quantum-mechanical (QM) basis methods, ${ }^{4-6}$ involves less traditional approaches, such as complex-space trajectory dynamics in real time ${ }^{7,8}$ and real-space trajectory dynamics in imaginary time.${ }^{9-11}$ Here we will consider how the wavefunction representation affects the character of the imaginary-time quantum trajectory dynamics and the accuracy of its numerical implementation.

The connection between the QM Hamiltonian evolution and the Boltzmann evolution via the transformation between the real and imaginary times (the Wick rotation ${ }^{12}$ ) was used by Feynman in the path integral formulation of statistical mechanics, ${ }^{13}$ and in a semiclassical context by Miller ${ }^{14}$ who related the Boltzmann evolution to the real-time dynamics on the inverted classical potential. The Boltzmann evolution of a wavefunction according to the diffusion equation with the QM Hamiltonian $\hat{H}$,

$$
\hat{H} \psi(x, \tau)=-\hbar \frac{\partial}{\partial \tau} \psi(x, \tau),
$$

is equivalent to Eq. (1) where the real time-variable $t$ is replaced by the imaginary time $\tau, t \rightarrow-\imath \tau$.

As $\tau \rightarrow \infty$, any initial wavefunction will evolve into the lowest energy eigenfunction (of the same symmetry if the system has a defined symmetry), since the lowest energy component is the slowest to decay - a feature behind the largest

\footnotetext{
a)Electronic mail: sgarashc@mail.chem.sc.edu.
}

exact QM calculations of the zero-point energies (ZPEs) using the diffusion Monte Carlo. ${ }^{15-19}$ Thus, for any initial $\psi$ the wavefunction energy $E$ converges to the $\mathrm{ZPE}$ value, $E_{0}$, in the course of evolution,

$$
E(\tau)=\frac{\langle\psi|\hat{H}| \psi\rangle_{\tau}}{\langle\psi \mid \psi\rangle_{\tau}}, \quad \lim _{\tau \rightarrow \infty} E(\tau)=E_{0} .
$$

The zero of energy scale has to be chosen so that $E_{0}>0$ to avoid the exponential growth of the wavefunction norm, $N(\tau)=\langle\psi \mid \psi\rangle_{\tau}$. The imaginary time evolution is also encountered in direct calculations of the thermal reaction rate constants $^{20}$ involving evaluation of the Boltzmann operator, ${ }^{21}$ $\exp (-\beta \hat{H})$, where $\tau \equiv \beta=\left(k_{B} T\right)^{-1}, T$ being the system temperature.

Recasting of the Schrödinger equation in terms of trajectories whether in real time, Eq. (1), or in imaginary time, Eq. (2), starts with representing all or part of a wavefunction using an exponent of the "phase" or "action" function $S$ to define the quantum trajectory momenta,

$$
p=\nabla S .
$$

The formalism below is given for a particle of mass $m$ in one Cartesian dimension $x$; multidimensional generalizations can be found in Refs. 22 and 23. For clarity, $\nabla$ is used to denote spatial derivatives, including the one-dimensional case $\nabla=\partial / \partial x$.

The real-time quantum or Bohmian trajectory formalism, ${ }^{1-3}$ for which the theory and implementations are described in detail in Ref. 24, is derived from the polar representation of a wavefunction,

$$
\psi^{\text {Bohm }}=A(x, t) \exp \left(\frac{l}{\hbar} S(x, t)\right),
$$


with $A(x, t)$ and $S(x, t)$ assumed to be real functions. This particular choice of representation is justified by the physical meaning of the evolution equations following from Eqs. (1), (4), and (5) in the Lagrangian frame-of-reference,

$$
\frac{d}{d t}=\frac{\partial}{\partial t}+\frac{p}{m} \frac{\partial}{\partial x} \text {. }
$$

These evolution equations are: (i) the continuity equation for the probability density, which contains all the terms proportional to $l$ and linear in $\hbar$,

$$
\frac{d A^{2}}{d t}=-\frac{\nabla p}{m} A^{2}
$$

and (ii) the classical-like equations of trajectory motion,

$$
\begin{gathered}
\frac{d S}{d t}=\frac{p^{2}}{2 m}-(V+U), \\
\frac{d x}{d t}=\frac{p}{m}, \quad \frac{d p}{d t}=-\nabla(V+U) .
\end{gathered}
$$

All QM effects stem from the non-local quantum potential $U$,

$$
U=-\frac{\hbar^{2}}{2 m} \frac{\nabla^{2} A}{A},
$$

which formally vanishes in the classical limit $\hbar \rightarrow 0$ or $m \rightarrow \infty$ inviting approximate and semiclassical implementations of the formalism. ${ }^{25-29}$ For numerical implementation, a system of equations (7)-(10) is solved, exactly or approximately, as reviewed in Ref. 24. Despite the conceptual appeal, however, for general classical potentials and wavefunctions, $U$ of Eq. (10) becomes singular near wavefunction nodes $(A(x, t)=0)$, or whenever QM interference is present, giving rise to unstable trajectory dynamics. To alleviate the ensuing difficulties of numerical implementation the Eulerian and arbitrary Lagrangian/Eulerian grids have been used ${ }^{30-33}$ to prevent the trajectories, i.e., the time-dependent grid points, from moving into the node region. The strategy helps with numerical stability, but does not change the singular character of underlying quantum trajectory dynamics, which is traceable back to the polar representation given in Eq. (5). The bipolar wavefunction representation has the capability of reproducing QM interference via superposition of multiple subwavepackets, ${ }^{34,35}$ but is more complicated in concept and in practice (especially in high dimensions).

The method of Bohmian trajectories with complex action (BOMCA) (Ref. 10) is based on the representation of a wavefunction simpler than Eq. (5),

$$
\psi^{\text {ВОМСА }}=\exp \left(\frac{l}{\hbar} S(x, t)\right),
$$

substituted into Eq. (1). The BOMCA trajectory dynamics defined in the Lagrangian frame given by Eq. (6) unfolds in complex $(x, p)$ space. It captures QM interference via multiple complex-space paths contributing to $\psi(x, t)$ evaluated at a single real $x$, thus, avoiding the node problem. However, from the numerical point-of-view BOMCA is very challenging due to analytical continuation of all quantities into the complex plane and due to the initial/final value search of the complex-space trajectories contributing to the wavefunction on real $x .^{8,10,36}$

\section{QUANTUM TRAJECTORY DYNAMICS IN IMAGINARY TIME}

\section{A. Formalism}

In contrast to the real-time/complex-valued quantum trajectory dynamics of BOMCA, the diffusion equation (2), which is real since it does not mix real and imaginary parts of $\psi$, allows construction and investigation of different types of real-space trajectory dynamics. As shown by Liu and Makri, ${ }^{9}$ substitution of $t \rightarrow-l \tau$ in the original real-time Bohmian equations (5) and (9) results in a singular trajectory representation of a Gaussian wavepacket, a feature attributed to the non-uniqueness of representing $\psi(x, \tau)$ with the amplitude, $A(x, \tau)$, and "phase" factors, $\exp (-S(x, \tau) / \hbar)$. The repartitioning of $\psi(x, \tau)$ between these two factors was successfully introduced to generate non-singular and, in fact, nearly stationary trajectories enabling first multidimensional ZPE calculations from the imaginary-time Bohmian trajectories. The approach was implemented using the "independent" trajectory approach ${ }^{37}$ based on the truncated hierarchy of equations for $S, A$, and their high-order derivatives.

Perhaps, the simplest route to the imaginary-time quantum trajectory dynamics begins with the positive wavefunction expressed as a single exponential function,

$$
\psi(x, \tau)=\exp (-S(x, \tau) / \hbar) .
$$

Note that since Eq. (2) is real, the imaginary and real parts of an initially complex wavefunction do not mix and can be propagated independently. Thus, without loss of generality, we take $\psi(x, \tau)$ to be real. In addition, for $S(x, 0)$ to be smooth, we take $\psi(x, 0)$ to be nodeless. Substitution of Eq. (12) into Eq. (2) (followed by division by $\psi(x, \tau)$ ) gives the following Hamilton-Jacobi equation:

$$
\frac{\partial S}{\partial \tau}=-\frac{(\nabla S)^{2}}{2 m}+V+\frac{\hbar}{2 m} \nabla^{2} S .
$$

Defining the momentum according to Eq. (4), the last term in Eq. (13) is interpreted as the momentum-dependent quantum potential (MDQP), ${ }^{11,20}$

$$
U(x, \tau)=\frac{\hbar \nabla p}{2 m} .
$$

In the Lagrangian frame, Eq. (13) gives quantum trajectory dynamics on the inverted classical potential with MDQP of Eq. (14) added to it,

$$
\begin{gathered}
\frac{d S}{d \tau}=\frac{p^{2}}{2 m}+V+U, \\
\frac{d x}{d \tau}=\frac{p}{m}, \quad \frac{d p}{d \tau}=\nabla(V+U) .
\end{gathered}
$$

For practicality of multidimensional implementation, we compute MDQP of Eq. (14), which formally vanishes in the classical limit (as was the case for the quantum potential in real time) approximately from the global least squares fit $^{38}$ to $p$ in the Taylor basis $\vec{f}=\left(1, x, x^{2} \ldots\right)$. The optimal expansion coefficients $\vec{c}$, minimizing $\left\langle(p-\vec{f} \cdot \vec{c})^{2}\right\rangle$, are found from the system of linear equations,

$$
\mathbf{M} \vec{c}=\vec{b}, \quad \mathbf{M}=\langle\vec{f} \otimes \vec{f}\rangle, \quad \vec{b}=\langle p \vec{f}\rangle .
$$


The linear basis is exact for Gaussian wavefunctions (for the harmonic potentials) and produces zero quantum force. The quadratic basis is the smallest one affecting trajectory dynamics. The expectation values are evaluated according to Eq. (18). Equation (17) will be used to approximate MDQP (and other terms containing spatial derivatives of $p$ ) needed in the Eulerian and in the modified Lagrangian dynamics described below.

Besides the classical-like appearance of the MDQP equations (15) and (16), in the Lagrangian frame-of-reference the contribution of the quantum potential of Eq. (14) to expectation values is cancelled by the time dependence of the volume element, $\delta x_{\tau}$, associated with each trajectory $\left(x_{t}, p_{t}\right),{ }^{11}$

$$
\delta x_{\tau}=\delta x_{0} \exp \left(\int_{0}^{\tau} \frac{\nabla p_{t}}{m} d t\right) .
$$

For an operator $\hat{\Omega}$ and the wavefunction (12), the change of integration variable from coordinate $x$ to the time-dependent trajectory positions $x_{\tau}$ gives

$$
\langle\Omega\rangle=\int \Omega(x, \tau) e^{-2 S(x, \tau)} d x=\int \Omega\left(x_{\tau}\right) e^{-2 S\left(x_{\tau}\right)} d x_{\tau} .
$$

The action function computed along the trajectory $\left(x_{t}, p_{t}\right)$ from Eq. (15) is

$$
S\left(x_{\tau}\right)=S\left(x_{0}\right)+\int_{0}^{\tau}\left(\frac{p_{t}^{2}}{2 m}+V\left(x_{t}\right)+\frac{\nabla p_{t}}{2 m}\right) d t .
$$

The last term cancels the time dependence of $\delta x_{\tau}$ in the "trajectory-specific" probabilities, $P\left(x_{\tau}\right)$ $=\exp \left(-2 S\left(x_{\tau}\right)\right) \delta x_{\tau}$, contributing to the expectation values. Discretizing the wavefunction via a trajectory ensemble, the expectation value becomes

$$
\begin{aligned}
\langle\Omega\rangle & =\int \Omega\left(x_{\tau}\right) \exp \left(-2 \int_{0}^{\tau}\left(\frac{p_{t}^{2}}{2 m}+V\left(x_{t}\right)\right) d t\right) \delta x_{0} \\
& =\sum_{j} \Omega\left(x_{\tau}^{(j)}\right) \exp \left(-2 \tilde{S}_{\tau}^{(j)}\right) w^{(j)} .
\end{aligned}
$$

In Eq. (18), index $j$ labels trajectories; the trajectory weight $w^{(j)}$ denotes the initial contribution of the $j$ th trajectory to the average,

$$
w^{(j)}=\exp \left(-2 S\left(x_{0}^{(j)}\right)\right) \delta x_{0}^{(j)} .
$$

$\tilde{S}_{\tau}$ denotes the "classical" part of the action function computed along the quantum (influenced by the classical and quantum potentials) trajectory,

$$
\tilde{S}_{\tau}=\int_{0}^{\tau}\left(\frac{p_{t}^{2}}{2 m}+V\left(x_{t}\right)\right) d t .
$$

The MDQP formulation given by Eqs. (12), (15), and (16) has been shown ${ }^{11,20}$ to give accurate ZPE estimates for anharmonic systems, including the double well, and for the triatomic molecules with a reasonably small (quadratic) fitting basis determining $U$ and to converge to the QM result for larger bases (3-6 functions). For multidimensional bound systems, however, the sampling, or the trajectory representation problem was identified: in bound classical potentials $(-V$ is a barrier) the Lagrangian trajectories fall off the barrier top and leave the region of high wavefunction density. Consequently, to represent a wavefunction at long times, we needed several thousand randomly distributed at $\tau=0$ trajectories already in three dimensions, even when introducing importance sampling that emphasized low energy region of $V$.

To avoid the sampling (or representation) problem in the ZPE calculations, evolution in the Eulerian frame appears as a logical alternative to the Lagrangian evolution. Since the ZPE is determined by the energy eigenstate, which is a stationary object, the fixed-in-time points placed in the lowenergy regions of the potential will remain in the areas of high ground state density, and thus will be adequate for the description of the ground state wavefunction (and of a wavefunction decaying to it) at all times. To determine the evolving wavefunction at stationary points, instead of the Lagrangian equations (15) and(16), functions $S$ and $p$ are evolved in the Eulerian frame according to Eq. (13) and its gradient,

$$
\frac{\partial p}{\partial \tau}=-\frac{p \nabla p}{m}+\nabla(V+U) .
$$

Instead of the quantum trajectories, one initializes random grid-points fixed in space; for each point function $p$ is defined by Eq. (4) as before. In Ref. 7, the independent "zero-velocity" trajectories were invoked to implement the imaginary-time Hamiltonian-Jacobi equation and high-order gradients of $S$ in one dimension. For reasons of scalability, we implement the imaginary-time Eulerian evolution of $S$ and $p$ once again approximately, computing $\nabla p$ and $\nabla^{2} p$ on the right-hand side of Eqs. (13), (14), and (20) from the global least squares fit to $p$ given by Eq. (17).

\section{B. Comparison of the approximate MDQP dynamics in the Eulerian and Lagrangian frames of reference}

Let us start with an illustration of the MDQP dynamics performed in the Eulerian frame for a system of dimensionality $N_{\text {dim }}$ composed of linearly coupled harmonic oscillators. The potential,

$$
V=\frac{1}{2} \vec{x} \cdot \mathbf{A} \cdot \vec{x},
$$

is defined by the symmetric positive definite matrix $\mathbf{A}$. The diagonal elements of the matrix are taken as $A_{i i}=1$; the offdiagonal elements, set to a constant value, $A_{i i \pm 1}=\gamma$, change the mode frequencies of the system; the remaining matrix elements are set to zeros. All particles have unit mass: $m_{i}=1$, where $i=1 \ldots N_{d i m}$. For this potential, regardless of the offdiagonal elements, evolution of a wavefunction defined at $\tau=0$ as a multidimensional Gaussian function is exact for the linear basis $\vec{f}$ (of the size $N_{b}=N_{d i m}+1$ ) used to fit the components of $\vec{p}(\vec{x})$. The initial wavefunction is a direct product of Gaussian wavefunctions,

$$
\psi(x, 0)=\left(\frac{2 a}{\pi}\right)^{1 / 4} \exp \left(-a\left(x-x_{0}\right)^{2}\right)
$$

for each dimension. The parameter values listed in Table I describe the ground state with zero coupling, $\gamma=0$, for $N_{\text {dim }}$ $=40$. We have considered the coupling constants $\gamma=0.2$ and 
TABLE I. The zero-point energy from the Eulerian quantum trajectory dynamics for a system of 40 coupled harmonic oscillators. The coupling constant $\gamma$ (the first column) defines the ratio of the eigen-energies (the second column). $E_{0}^{\mathrm{QM}}$ is the analytical ZPE; $E_{0}^{\mathrm{MDQP}}$ is given by Eq. (3); $\bar{\varepsilon}=\sum_{k} \varepsilon\left(\vec{x}_{k}\right) / N_{\text {traj }}$ is the average energy value for the trajectory ensemble at the final time. The bottom row contains initial wavepacket parameters, final propagation time, and time step in a.u.

\begin{tabular}{lccccc}
\hline \hline$\gamma$ & $w_{40} / w_{1}$ & $N_{\text {traj }}$ & $E_{0}^{\mathrm{QM}}$ & $E_{0}^{\mathrm{MDQP}}$ & $\bar{\varepsilon}$ \\
\hline 0.2 & 1.53 & 50 & 19.7973 & 19.7972 & 19.7975 \\
0.4 & 2.98 & 100 & 19.0537 & 19.0535 & 19.0584 \\
$x_{0}=0 \mathrm{bohr}$ & \multicolumn{2}{c}{$a=0.5 \mathrm{bohr}^{-2}$} & $\tau=5.0$ & $d \tau=0.005$ \\
\hline \hline
\end{tabular}

0.4. These values define the Hamiltonians for which the ratio of the highest to the lowest mode frequencies are 1.53 and 2.98 , respectively, so that our $\psi(\vec{x}, 0)$ is noticeably different from the ground state of the coupled system. The ZPEs are obtained with five-digit accuracy from dynamics of an ensemble of 50-100 points as shown in Table I. Convergence to the ZPE value despite very sparse sampling is explained by the fact that energies of individual trajectories,

$$
\varepsilon(\vec{x})=\psi^{-1}(\hat{H} \psi)=-\frac{\vec{p} \cdot \vec{p}}{2 m}+V+\frac{\hbar \nabla \cdot \vec{p}}{2 m},
$$

become essentially the same with time as shown in Fig. 1. Consequently, the wavefunction norm cancels in the normalized energy expression of Eq. (3). Representation of a wavefunction itself or estimates of quantities involving higher moments over the trajectory distributions, such as energies of the excited states, require about 10000 trajectories as seen from Table II.

The low-lying excited energy levels were obtained as the generalized eigenvalues of the Hamiltonian evaluated in a small basis, such as the Taylor fitting basis $\vec{f}$ multiplied by $\psi(x, \tau)$,

$$
\mathbf{H} \psi_{n}=E_{n} \mathbf{M} \psi_{n} .
$$

(The approximate MDQP evolution with projections, a more complicated approach, was used to evaluate the excited states in a double well. ${ }^{11}$ ) As shown in Appendix A, the Hamilto-

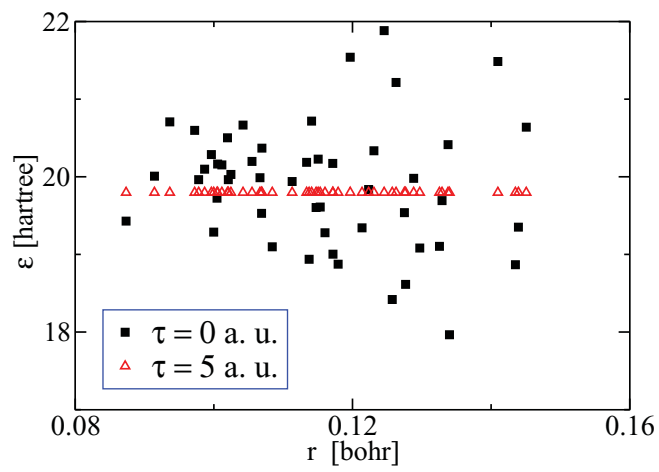

FIG. 1. The Eulerian imaginary-times evolution for 40 coupled harmonic oscillators. The initial (squares) and final (triangles) energy of the points, $\varepsilon$ of Eq. (23), are shown for $\gamma=0.2$ as a function of the average distance from the center of the well per dimension, $r=|\vec{x}| / N_{\text {dim }}$.
TABLE II. Selected energy levels $(0 \leq \mathbf{n} \leq 15)$ for a system of 15 linearly coupled harmonic oscillators. Data in rows 1-3 are obtained after imaginarytime evolution to $\tau=4.0$ a.u. for which the central processing unit (CPU) time is listed. The last row contains eigenvalues obtained in the linear basis at $\tau=0.0$

\begin{tabular}{lcccccccc}
\hline \hline$N_{\text {traj }} / 10^{3}$ & $\mathrm{CPU}[\mathrm{s}]$ & $\mathbf{n}=0$ & 1 & 2 & 7 & 8 & 14 & 15 \\
\hline 2.5 & 4.96 & 7.4273 & 8.1660 & 8.1969 & 8.3920 & 8.4262 & 8.6149 & 8.6406 \\
25 & 52.1 & 7.4273 & 8.1932 & 8.2202 & 8.3893 & 8.4460 & 8.5945 & 8.6079 \\
125 & 259.5 & 7.4273 & 8.2077 & 8.2244 & 8.3879 & 8.4212 & 8.5951 & 8.5990 \\
$\mathrm{QM}$ & Analyt. & 7.4273 & 8.2069 & 8.2213 & 8.3875 & 8.4273 & 8.5976 & 8.6073 \\
2.5 & $\tau=0$ & 7.5088 & 8.2677 & 8.3055 & 8.4748 & 8.5091 & 8.7081 & 8.7390 \\
\hline \hline
\end{tabular}

nian matrix elements in Eq. (24) are

$$
H_{i j}=\left\langle f_{i}|\varepsilon| f_{j}\right\rangle+\frac{\hbar}{2 m}\left\langle\nabla f_{i} \mid \nabla f_{j}\right\rangle .
$$

The overlap matrix $\mathbf{M}$ is defined in Eq. (17).

The low-lying excited state energies for a system of 15 linearly coupled oscillators, defined by Eq. (21) for $\gamma=0.2$ are shown in Table II. The limited (linear) basis $\vec{f}$ gives more accurate estimates of eigenstates if $\psi(\vec{x}, \tau)$ is close to the ground state. Generally, Eq. (24) can be defined in a basis different from the fitting basis of the MDQP approximation and solved for several values of $\tau$ during the propagation to monitor the convergence of eigenvalues with time. If the bases in Eqs. (17) and (24) are the same, then estimation of the excited states requires little effort in addition to the approximate MDQP evolution.

Our application of MDQP dynamics in the Eulerian frame to the high-dimensional system of coupled harmonic oscillators shows that the approach is feasible and scales linearly with the number of trajectories. However, since a general multidimensional Gaussian evolving in a quadratic potential remains a Gaussian, the linear fitting basis gives correct functional form of $\vec{p}$. The fitting procedure is exact within the Monte Carlo integration error responsible for the discrepancies between the analytical and numerical results in Table II. Thus, this application does not illustrate the approximate MDQP regime analyzed in the remainder of this section. Before proceeding, we note that the earlier ZPE calculation for two coupled Morse oscillators mimicking $\mathrm{H}_{2}$ bond in the Eulerian frame was quite efficient $;^{20}$ the quadratic fitting gave the ZPE accurate within $0.5 \%$ in units of the harmonic ZPE value and overestimated the $\mathrm{ZPE}$ shift due to anharmonicity by a third of the exact QM result.

To start analyzing the accuracy of the approximate MDQP approach, we notice that there are two differences between the Eulerian and Lagrangian dynamics. (i) The Eulerian "trajectories" give efficient ground-state representation only if the ground state is localized and the trajectories are placed near the global minimum of $V$, whereas the Lagrangian trajectories explore the potential as they move. (ii) To implement the Eulerian evolution, the gradient of the momentum in the term $p \nabla p / m$ of Eq. (20) is approximated in addition to the MDQP present in both, Lagrangian and Eulerian formulations. This term is not an $\hbar$-quantity and therefore generally does not vanish in the classical limit. 
TABLE III. The zero-point energy of the quartic oscillator from the approximate MDQP trajectory evolution up to $\tau=2.0$ a.u. Asterisks mark the values that are not converged with respect to $\tau$. The exact and harmonic ZPE values for the system are 0.804 and 0.5 hartree, respectively. Results for the Eulerian evolution with exact $\nabla p$ as described in text are given in the last column.

\begin{tabular}{|c|c|c|c|c|c|c|}
\hline \multirow{2}{*}{$\begin{array}{l}\text { Dynamics } \\
N_{t r a j} \\
N_{b}\end{array}$} & \multicolumn{2}{|c|}{$\begin{array}{l}\text { Lagrangian } \\
1001\end{array}$} & \multicolumn{2}{|c|}{$\begin{array}{c}\text { Eulerian } \\
101\end{array}$} & \multicolumn{2}{|c|}{$\begin{array}{c}\text { Eulerian }^{\nabla p} \\
101\end{array}$} \\
\hline & $E$ & $d E / d \tau$ & $E$ & $d E / d \tau$ & $E$ & $d E / d \tau$ \\
\hline 2 & 0.742 & $-3 \times 10^{-3}$ & $0.009 *$ & -0.51 & 0.794 & $-7 \times 10^{-5}$ \\
\hline 4 & 0.804 & $2 \times 10^{-5}$ & $0.578^{*}$ & -0.18 & 0.804 & $-1 \times 10^{-6}$ \\
\hline 6 & 0.804 & $3 \times 10^{-6}$ & 0.803 & $-7 \times 10^{-4}$ & 0.804 & $-1 \times 10^{-7}$ \\
\hline
\end{tabular}

To quantify these differences, let us examine a strongly anharmonic one-dimensional potential,

$$
V=\frac{x^{2}}{2}+x^{4}
$$

for a particle mass $m=1$, studied, for example, in Refs. 9 and 11. The initial wavefunction defined by Eq. (22) and parameters $a=0.5 \mathrm{bohr}^{-2}$ and $x_{0}=0$ describe the ground state of the harmonic part of $V$. The initial positions of the quantum trajectories are taken on a uniform grid, their initial momenta are $p=2 a\left(x-x_{0}\right)$. The momentum was fitted using the Taylor bases through fifth order, $N_{b}=2,4$, and 6. Evolution was performed up to $\tau=2.0$ a.u. At this time, the wavefunction energy for calculations converged with respect to $N_{b}$, reached plateau values. One thousand trajectories initially spaced by 0.006 bohr and 100 points space by 0.06 bohr were used for the Lagrangian and Eulerian evolutions, respectively. The wavefunction energies and their convergence to the ZPE values are given in Table III.

While both, the Lagrangian and the Eulerian formulations, give ZPE values of the same accuracy for $N_{b}=6$ (when $p$ is fitted with high accuracy), the low-order polynomial fittings show significant differences. The linear basis fit generating zero quantum force yields unphysical energies at the end of the Eulerian evolution; at the same time the significant part of the ZPE change due to anharmonicity (79\%) is captured in the Lagrangian implementation. (The exact ground state energy for the system is $E_{0}=0.804$ hartree. The harmonic ZPE value is 0.5 hartree.) The wavefunction energy of the correct order of magnitude, though not reaching a plateau value with time, is achieved in the Eulerian formulation only for the cubic fitting basis, $N_{b}=4$, whereas the Lagrangian formulation already gives a well-converged result for the same basis. To verify that the source of inferior performance of the Eulerian formulation is, indeed, the $p \nabla p / m$ term of Eq. (13), which was incorporated exactly in the Lagrangian frame, we combined accurate $\nabla p$ evaluation by finite difference with the MDQP evaluated from the global polynomial fitting of $p$. The obtained ZPE values were similar in accuracy to the Lagrangian ZPE for the same fitting basis size. For a potential with quartic anharmonicity $V^{(4)}=k x^{4}$, a simple error analysis shows that after incrementing all quantities by a single time step $t$ from their initial values and fitting the resulting $p(t)$ with a polynomial in $x_{t}$ for the Lagrangian frame and in $x$ for the Eulerian frame, the lowest (cubic) fitting coefficient due to anharmonicity is smaller for the Lagrangian frame,

$$
c_{3}^{\text {Eul }}=4 k t, \quad c_{3}^{\text {Lagr }}=\frac{4 k t}{(1+t a / m)^{3}} .
$$

We also tried evolution with the momentum gradients described in Appendix B, expecting that evolution of $\nabla p$ for each quantum trajectory in the ensemble would improve the accuracy of the Eulerian formulation implemented in a small fitting basis. We found that for the lowest order fitting, giving zero approximate quantum force, this was not the case: there was no improvement on the unphysical values of the ZPE estimate in the Eulerian formulation. For the next basis size considered, the accuracy was improved by a factor of 2 , but was still worse than for the Lagrangian dynamics with the same fitting basis. This also points to the importance of treating the $p \nabla p / m$ term accurately.

\section{MODIFICATIONS OF THE LAGRANGIAN QUANTUM TRAJECTORY FORMULATION}

Our experience with the Eulerian frame can be summarized as follows. While the stationary trajectories have important advantages for the ground state calculations - (i) trajectories started in the low energy region continue to contribute to the ground state at later times allowing sparse sampling in high-dimensional space and (ii) the classical potential $V$ has to be evaluated only once, which gives big computational savings for on-the-fly calculations - the small-basis fitting is accurate only for mildly anharmonic systems because a non- $\hbar$ term is approximated. The Lagrangian dynamics, which approaches classical dynamics as $\hbar \rightarrow 0$, gives more stable and accurate ZPE estimates. Therefore, we will reformulate the Lagrangian dynamics to reduce divergence of trajectories by changing the potential acting on the trajectories, rather than by simply postulating $d x / d \tau=0$ which is effectively done in the Eulerian formulation.

The single function in the exponent in Eq. (12) is the simplest representation of a real nodeless wavefunction, but it is not a unique one. Ideally, we want a formulation where the Lagrangian trajectories describing ground states do not move, at least for the quadratic potentials. This can be achieved by introducing the time-independent function $S_{0}(x)$ into $\psi$,

$$
\psi(x, \tau)=\exp \left(-S_{0}(x)-S(x, \tau) / \hbar\right) .
$$

The trajectory momentum is $p=\nabla S$ as given by Eq. (4) and used in all types of dynamics discussed here. Substitution of Eq. (28) into Eq. (2) and division by $\psi(x, \tau)$ in the Lagrangian frame of Eq. (6) give

$$
\frac{d S(x, \tau)}{d \tau}=\frac{p^{2}}{2 m}+V+U+V_{s}+V_{c},
$$

where $U$ is the MDQP given by Eq. (14). The stationary potential $V_{s}$ is defined by the time-independent analytical function $S_{0}$,

$$
V_{s}=\frac{\hbar^{2}}{2 m}\left(\nabla^{2} S_{0}-\left(\nabla S_{0}\right)^{2}\right)
$$


Term $V_{c}$ couples the time-dependent and time-independent components,

$$
V_{c}=-\frac{\hbar p \nabla S_{0}}{m}
$$

The term $\nabla p$ needed for evaluation of $\nabla V_{c}$ (an $\hbar / m$ quantity) will be determined from the global fitting of $p$ along with the MDQP, approximately. The gradient of Eq. (29) leads to the following equations of motion:

$$
\frac{d p}{d \tau}=\nabla\left(V+U+V_{s}+V_{c}\right), \quad \frac{d x}{d \tau}=\frac{p}{m} .
$$

The most efficient representation of the known ground state, $\psi_{0}(x)$, evolving in time is based, of course, on the separation of time and space variables,

$$
\begin{gathered}
S_{0}(x)=-\ln \psi_{0}(x), \\
S(x, \tau)=S(\tau)=S(0)+E_{0} \tau .
\end{gathered}
$$

In this case, $V_{s}$ of Eq. (30) cancels the classical potential $V$ up to a constant $E_{0}$ and the initial trajectory momenta are zeros. Therefore, the coupling potential $V_{c}$ and the total force acting on the trajectories are equal to zeros and remain so at later times: the trajectories are stationary and the only time dependence is in the function $S$ of Eq. (34).

In practice, $\psi_{0}(x)$ is not known and we choose $S(x, 0)$ and $S_{0}(x)$ as quadratic functions: $S_{0}(x)$ defines a timeindependent Gaussian localized where $\psi_{0}$ is presumed to be significant; $S(x, \tau)$ captures the rest of space and time dependence of $\psi(x, \tau)$ as it decays into the ground state. The trajectory spreading is reduced in two ways. (i) The quadratic function, $S_{0}=a_{0} x^{2}$, generates a parabolic barrier $V_{s}$ which counteracts the divergence due to a classical well $V$. (ii) For the same Gaussian initial wavefunction of width $a$ (Eq. (22)), the representation given by Eq. (28) results in smaller initial trajectory momenta, $p=2\left(a-a_{0}\right) x$, compared to the momenta of the original single-function representation given by Eq. (12). The choice of $a$ and $a_{0}$ should be guided by the normal mode frequencies, so that the total wavefunction $\psi(x, 0)$ is more localized than the eigenstate and $a_{0}, a_{0}<a$, defines a Gaussian more delocalized than the eigenstate to avoid convergent trajectory dynamics that is unphysical and will lead to numerical problems.

The illustration below is given for the quartic potential of Eq. (26): 100 trajectories spaced by 0.04 bohr uniformly sample the initial wavefunction given by Eq. (22) for $a=0.5 \mathrm{bohr}^{-2}$. The propagation was performed up to $\tau=2.0$ a.u. for $a_{0}=0.25$ and $0.5 \mathrm{bohr}^{-2}$ and compared to the original MDQP setup corresponding to $a_{0}$ $=0$. The least squares fit of $p$ with the cubic polynomial is analogous to Eq. (17) and determines all the necessary spatial derivatives of $p$ in the equations of motion. The divergence of trajectories with time (Fig. 2(a)) is reduced as we go from $a_{0}=0$ to $a_{0}=0.5$ bohr $^{-2}$. The effect of the dynamics modification is clearly seen in the description of the wavefunction (Fig. 2(b)): plotting only trajectories with the wavefunction density above $10^{-16}$ one has 5,11 , and 23 points at the end of propagation for $a_{0}=0,0.25$, and $0.5 \mathrm{bohr}^{-2}$, respectively. Convergence
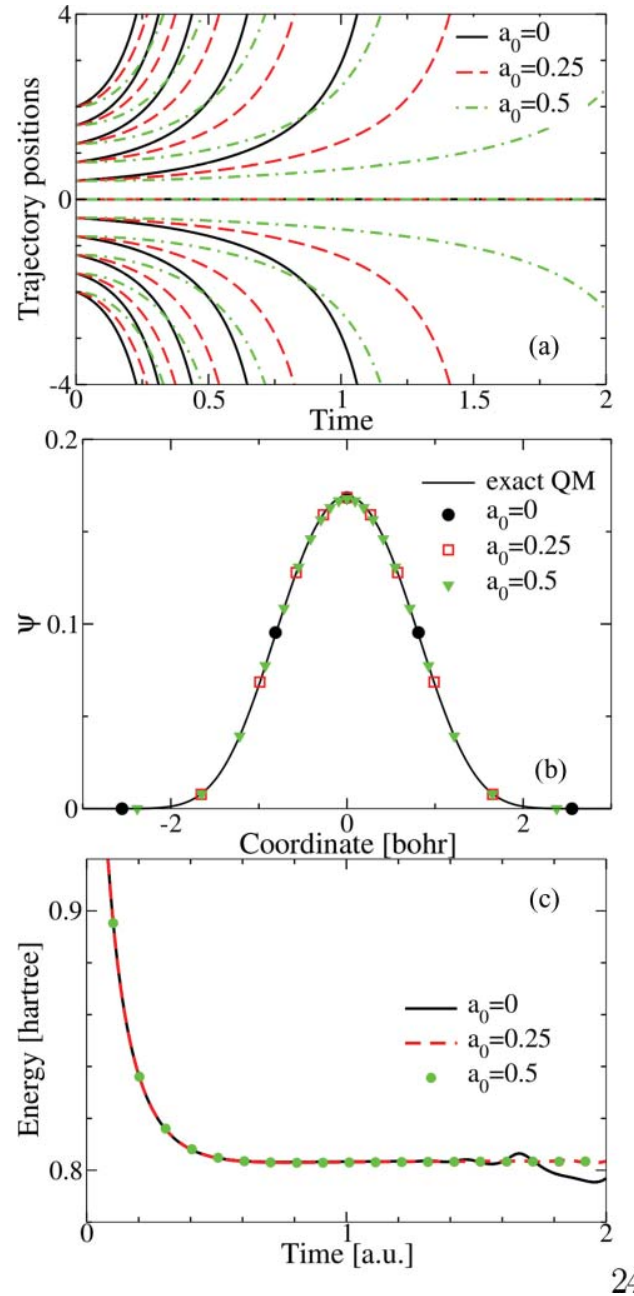

24

FIG. 2. Modification of the Lagrangian dynamics in the quartic well. The considered stationary width values are $a_{0}=0,0.25$, and $0.5 \mathrm{bohr}^{-2} ; \psi(x, 0)$ is given by Eq. (22) for $a=0.5 \mathrm{bohr}^{-2}$. (a) Selected trajectories; (b) $\psi(x, \tau)$ for $\tau=2$ a.u.; and (c) the wavefunction energy.

to the ZPE value is shown in Fig. 2(c): an ensemble of 100 quantum trajectories is enough to obtain converged $\mathrm{E}_{0}$ for $a_{0}=0.5 \mathrm{bohr}^{-2}$, which is not the case for $a_{0}=0$. After $\tau>1.5$ a.u., we observe oscillations of the wavefunction energy as the original Lagrangian trajectory representation of $\psi(x, \tau)$ becomes inadequate.

Similar to Ref. 9, the wavefunction representation via $S_{0}$ and $S$ given by Eq. (28) can also be used to repartition $\psi(x, \tau)$ between the stationary and dynamic components without approximations. The function $\Delta$ corresponding to the fitted function $\tilde{p}$,

$$
\Delta=\int \tilde{p} d x+\langle S\rangle-\left\langle\int \tilde{p} d x\right\rangle,
$$

can be subtracted from the time-dependent "dynamic" component $S$ and added to the time-independent "stationary" function $S_{0}$, with the appropriate change in the trajectory momenta,

$$
\begin{aligned}
& S_{0}^{\text {new }}=S_{0}+\Delta \\
& S^{\text {new }}=S-\Delta \\
& p^{\text {new }}=p-\tilde{p} .
\end{aligned}
$$



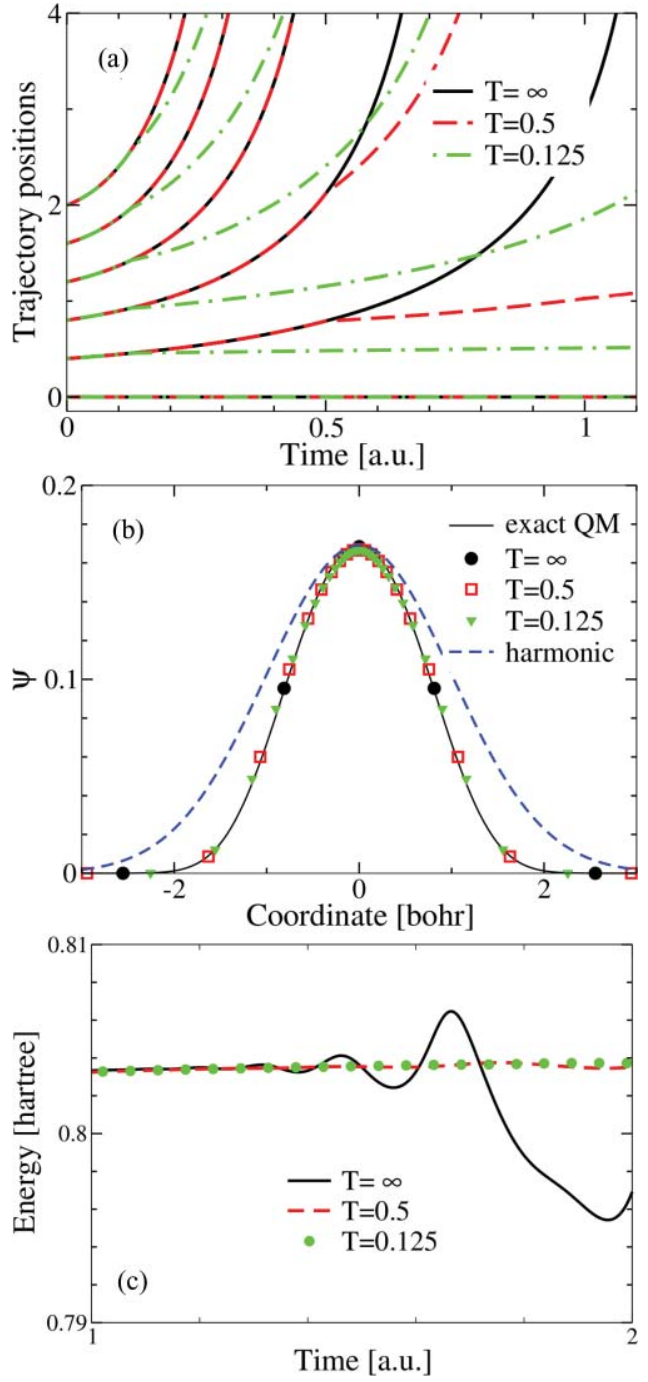

FIG. 3. Lagrangian dynamics with wavefunction repartitioning for $\psi(x, 0)$ given by Eq. (22). Solid lines mark the results without repartitioning on all panels. (a) Selected trajectories. (b) $\psi(x, \tau)$ for $\tau=2$ a.u. The ground state wavefunction for the harmonic oscillator, scaled to match the maximum of exact QM wavefunction, is shown with dash. (c) The wavefunction energy. Exact $\mathrm{ZPE}$ is $E_{0}=0.804$ hartree.

For the model system described in this section, the effect of repartitioning (Eq. (36)) on dynamics is found to be qualitatively similar to calculations with various $a_{0}$ described above. We defined $\Delta$ by the linear part of the cubic fit to $p$ so that $V_{s}$ remained parabolic in $x$ at all times. The results shown in Fig. 3 are obtained for initial $a_{0}=0$ with the wavefunction repartitioning at intervals of $T=0.5$ and 0.125 a.u. and compared to the calculation with no repartitioning. The remaining parameter values are the same as already described. As seen from Fig. 3(a) the repartitioning procedure resets the trajectory momenta to smaller values and introduces analytical $V_{s}$ reducing the trajectory divergence and improving wavefunction sampling. As shown in Fig. 3(b) at the end of propagation 21 and 33 trajectories (for $T=0.5$ and 0.125 a.u., respectively) contribute to the wavefunction compared to five trajectories for dynamics with no repartitioning. Better wavefunction representation improved the ZPE convergence shown in Fig. 3(c). Of course, non-zero $a_{0}$ and wavefunction reparti- tioning can be used in the same calculation: the goal is to balance efficient trajectory representation of the evolving wavefunction with the exploration of the classical potential with the quantum trajectories.

\section{SUMMARY}

We have analyzed the accuracy of the imaginary-time quantum trajectory evolution with the approximate MDQP in the Eulerian and Lagrangian frames of reference in the context of the ground state calculations. The problem of the Lagrangian quantum trajectory evolution is that the trajectories leave the region of localization of the ground state. We have concluded that while the Eulerian evolution has very appealing features - classical potential and force are evaluated only once and the coordinate space sampling points do not move - there is also a significant drawback: for strongly anharmonic systems the Eulerian approach is not as accurate as the Lagrangian quantum trajectory dynamics because it does not have classical dynamics as its $\hbar \rightarrow 0$ limit. The error is traced to the approximation of $\nabla p$ in the non- $\hbar$ term in the Eulerian formulation (Eqs. (13) and (20)), which is incorporated exactly in the Lagrangian formulation. To counteract the divergent dynamics of the Lagrangian trajectories at long $\tau$, we have introduced (i) a stationary component into the wavefunction form, Eq. (28). This modification generates analytical time-independent potential which reduces the total force acting on the trajectories and results in smaller initial momenta defined by the dynamic component. Both consequences improve the wavefunction sampling with trajectories. If the stationary function described the ground state, the new Lagrangian trajectories would become stationary. The stationary/dynamic wavefunction representation also allows (ii) repartitioning of $\psi(x, \tau)$ between the two components without approximations. This repartitioning resets the trajectory momenta to smaller values improving the wavefunction representation even further. Work-in-progress includes development of the criterion for a balanced application of the two modifications of the Lagrangian quantum trajectory dynamics and multidimensional chemical applications.

\section{ACKNOWLEDGMENTS}

This material is based upon work partially supported by the South Carolina Research foundation and by the National Science Foundation under Grant No. CHE-1056188.

\section{APPENDIX A: THE HAMILTONIAN MATRIX ELEMENTS}

In the context of the imaginary-time quantum-trajectory dynamics the low-lying excited eigenstates can be determined as the generalized eigenvalues of the Hamiltonian matrix $\mathbf{H}$ as given by Eq. (24). The matrix elements are evaluated in a basis of functions $\vec{f}(\vec{x})$, multiplied by the time-dependent wavefunction, $\psi(\vec{x}, \tau)$. For sufficiently long $\tau$, the initially nodeless wavefunction $\psi(\vec{x}, \tau)=\exp (-S(\vec{x}, \tau))$ approaches the ground state. The simplest basis describing - for anharmonic potentials approximately - one excitation per degree of freedom is linear, $\vec{f}=\left(x_{1}, x_{2}, \ldots, x_{N_{d i m}}, 1\right)$. Larger bases 
can be used to estimate higher eigenstates and/or for better accuracy. Using one spatial dimension and $\hbar=1$ for clarity, the Hamiltonian matrix elements are

$$
\begin{aligned}
H_{i j}= & \int e^{-S} f_{i}\left(V-\frac{\nabla^{2}}{2 m}\right) e^{-S} f_{j} d x \\
= & \int e^{-S} f_{i}\left(V f_{j} e^{-S}-\frac{f_{j}}{2 m} \nabla^{2} e^{-S}\right) d x \\
& -\frac{1}{2 m} \int e^{-S} f_{i} 2\left(\nabla e^{-S}\right)\left(\nabla f_{j}\right) d x \\
& -\frac{1}{2 m} \int e^{-S} f_{i} e^{-S}\left(\nabla^{2} f_{j}\right) d x .
\end{aligned}
$$

For infinite integration range, integration of the last integral in Eq. (A2) by parts gives

$$
\begin{aligned}
\int e^{-2 S} f_{i}\left(\nabla^{2} f_{j}\right) d x= & \left.e^{-2 S} f_{i}\left(\nabla f_{j}\right)\right|_{-\infty} ^{\infty}-\int e^{-2 S}\left(\nabla f_{i}\right)\left(\nabla f_{j}\right) d x \\
& -\int f_{i}\left(\nabla f_{j}\right)\left(\nabla e^{-2 S}\right) d x
\end{aligned}
$$

With that, assuming $\psi(x, \tau)$ vanishes at $\pm \infty$ and using definition $\nabla S=p$ in Eq. (A1), the expression for $H_{i j}$ becomes

$$
\begin{aligned}
H_{i j}= & \int_{\infty}^{\infty} e^{-2 S} f_{i} f_{j}\left(V-\frac{p^{2}}{2 m}+\frac{\nabla p}{2 m}\right) d x \\
& +\frac{1}{2 m} \int_{\infty}^{\infty} e^{-2 S} \nabla f_{i} \nabla f_{j} d x \\
= & \left\langle f_{i}|\varepsilon| f_{j}\right\rangle+\frac{1}{2 m}\left\langle\nabla f_{i} \mid \nabla f_{j}\right\rangle .
\end{aligned}
$$

Definitions (18) and (23) of the trajectory energies $\varepsilon$ and of the trajectory ensemble averages were used to obtain Eq. (A4).

\section{APPENDIX B: THE IMAGINARY-TIME EVOLUTION OF MOMENTUM GRADIENTS}

The real and imaginary time propagation of the derivatives (above the first order) of the wavefunction phase and amplitude was considered by several research groups attracted by the idea of independent quantum trajectories, as a way to deal with the node problem in Bohmian dynamics, to reduce the number of trajectories to one or very few and to give their methods semiclassical flavor. Thus, the derivative propagation method, ${ }^{39,40}$ BOMCA and semiclassical approximation with zero velocity trajectories, ${ }^{7,10}$ and Bohmian dynamics from trajectory stability properties ${ }^{9,37}$ have emerged. All of them are based on the hierarchy of equations obtained by successive differentiation of the Hamilton-Jacobi equations (8) and (13) truncated at some finite order, such as 4 th or 6th, with higher order derivatives set to zero. These independent quantum trajectory methods were successfully applied to several one-dimensional systems and to ZPE calculation of $\mathrm{H}_{2} \mathrm{O}$ and $\mathrm{SO}_{2} .{ }^{9}$ However, the high-order truncation strategies might have certain shortcomings. The truncated set of equations is not guaranteed to converge to QM result, and it is expensive in multiple dimensions due to the large number of high-order derivatives of $S$ and $A$ and due to high-order derivatives of the classical potential $V$ for realistic chemical systems.

Our goal is to capture the dominant effects of non-locality of quantum mechanics using the quantum trajectory ensemble and to do this in a practical manner. Thus, we considered evolution of equations of motion for the imaginary-time quantum trajectories only through the second order (momentum gradient) with higher order derivatives found approximately from the global least squares fit to $\nabla p$. The second order scheme requires the gradients and the Hessians of $V$, which is typical for semiclassical methods, such as, for example, the HermanKluk propagator and frozen Gaussians. ${ }^{41,42}$ We have implemented the momentum gradient approach in the Lagrangian and Eulerian formulations, with the expectation of achieving higher accuracy ZPE estimates within a small fitting basis because now we approximate $\nabla p$ rather than $p$, as was done in the approximate MDQP. The Eulerian formulation is obtained by taking the gradient of Eq. (20) which, denoting $\nabla p \equiv g$, gives the following equations of motion:

$$
\begin{aligned}
& \frac{\partial S}{\partial \tau}=-\frac{p^{2}}{2 m}+\frac{\hbar g}{2 m}+V \\
& \frac{\partial p}{\partial \tau}=-\frac{p g}{m}+\frac{\hbar \nabla g}{2 m}+\nabla V \\
& \frac{\partial g}{\partial \tau}=-\frac{p \nabla g}{m}-\frac{g^{2}}{m}+\frac{\hbar \nabla^{2} g}{2 m}+\nabla^{2} V .
\end{aligned}
$$

The Lagrangian formulation is obtained by transforming Eq. (B1) into the Lagrangian frame given by Eq. (6).

To implement the quantum trajectory dynamics with Hessians given by Eq. (B1) approximately we performed the least squares fit of the momentum gradient, $g$, in terms of monomials, as described by Eq. (17) with $p$ replaced by $g$. Numerical studies of one-dimensional model systems of Ref. 11 (the Morse potential and the double well) in the Lagrangian frame showed that while there were instances when the momentum gradient fitting using $N_{b}$ functions yielded more accurate ZPEs (relative errors were twice smaller) than those obtained with the momentum fitting in a basis $N_{b}+1$, we also had counter examples. All-in-all, there was no obvious advantage to the more expensive dynamics with Hessians. Dynamics with Hessians for the quartic well of Sec. II B did not improve the accuracy of the Eulerian frame implementation, which supports the conclusion at the end of Sec. II B.

${ }^{1}$ D. Bohm, Phys. Rev. 85, 166 (1952).

${ }^{2}$ E. Madelung, Z. Phys. 40, 322 (1927)

${ }^{3}$ L. de Broglie, An Introduction to the Study of Wave Mechanics (E. P. Dutton and Company, New York, 1930).

${ }^{4}$ J. C. Light and T. Carrington, Jr., Adv. Chem. Phys. 114, 263 (2000).

${ }^{5}$ H. D. Meyer, U. Manthe, and L. S. Cederbaum, Chem. Phys. Lett. 165, 73 (1990).

${ }^{6}$ H. D. Meyer and G. A. Worth, Theor. Chem. Acc. 109, 251 (2003).

${ }^{7}$ Y. Goldfarb, I. Degani, and D. J. Tannor, J. Chem. Phys. 125, 231103 (2006)

${ }^{8}$ C.-C. Chou and R. E. Wyatt, J. Chem. Phys. 128, 234106 (2008).

${ }^{9}$ J. Liu and N. Makri, Mol. Phys. 103, 1083 (2005).

${ }^{10}$ Y. Goldfarb, I. Degani, and D. J. Tannor, Chem. Phys. 338, 106 (2007).

${ }^{11}$ S. Garashchuk, J. Chem. Phys. 132, 014112 (2010).

${ }^{12}$ P. Ramond, Field Theory: A Modern Primer (Addison-Wesley, Reading, MA, 1990).

${ }^{13}$ R. P. Feynman and A. R. Hibbs, Quantum Mechanics and Path Integrals (McGraw-Hill, New York, 1965). 
${ }^{14}$ W. H. Miller, J. Chem. Phys. 55, 3146 (1971).

${ }^{15}$ D. Blume, M. Lewerenz, P. Niyaz, and K. B. Whaley, Phys. Rev. E 55, 3664 (1997)

${ }^{16}$ D. M. Ceperley and L. Mitas, Monte Carlo Methods in Quantum Chemistry, Advances in Chemical Physics, Vol. 93 (Wiley, New York, 1996).

${ }^{17}$ W. A. Lester,Jr., L. Mitas, and B. Hammond, Chem. Phys. Lett. 478, 1 (2009).

${ }^{18}$ A. Viel, M. D. Coutinho-Neto, and U. Manthe, J. Chem. Phys. 126, 024308 (2007).

${ }^{19}$ C. E. Hinkle and A. B. McCoy, J. Phys. Chem. A 112, 2058 (2008).

${ }^{20}$ S. Garashchuk, Chem. Phys. Lett. 491, 96 (2010).

${ }^{21}$ W. H. Miller, S. D. Schwartz, and J. W. Tromp, J. Chem. Phys. 79, 4889 (1983).

${ }^{22}$ V. A. Rassolov, S. Garashchuk, and G. C. Schatz, J. Phys. Chem. A 110, 5530 (2006).

${ }^{23}$ S. Garashchuk and T. Vazhappilly, J. Chem. Phys. C 114, 20595-20602 (2010).

${ }^{24}$ R. E. Wyatt, Quantum Dynamics with Trajectories: Introduction to Quantum Hydrodynamics (Springer-Verlag, Berlin, 2005).

${ }^{25}$ S. Garashchuk and V. A. Rassolov, Chem. Phys. Lett. 364, 562 (2002).
${ }^{26}$ J. B. Maddox and E. R. Bittner, J. Phys. Chem. B 106, 7981 (2002).

${ }^{27}$ J. B. Maddox and E. R. Bittner, J. Chem. Phys. 119, 6465 (2003).

${ }^{28}$ A. Donoso, Y. J. Yeng, and C. C. Martens, J. Chem. Phys. 119, 5010 (2003).

${ }^{29}$ S. Garashchuk and V. A. Rassolov, J. Chem. Phys. 120, 1181 (2004).

${ }^{30}$ R. E. Wyatt and E. R. Bittner, J. Chem. Phys. 113, 8898 (2000).

${ }^{31}$ R. E. Wyatt, J. Chem. Phys. 117, 9569 (2002).

${ }^{32}$ C. J. Trahan and R. E. Wyatt, J. Chem. Phys. 118, 4784 (2003).

${ }^{33}$ B. K. Kendrick, J. Chem. Phys. 119, 5805 (2003).

${ }^{34}$ K. Park, B. Poirier, and G. Parlant, J. Chem. Phys. 129, 194112 (2008).

${ }^{35}$ B. Poirier, J. Theor. Comput. Chem. 6, 99 (2007).

${ }^{36}$ J. K. David and R. E. Wyatt, J. Chem. Phys. 128, 094102 (2008).

${ }^{37}$ J. Liu and N. Makri, J. Phys. Chem. A 108, 5408 (2004).

${ }^{38}$ W. H. Press, B. P. Flannery, S. A. Teukolsky, and W. T. Vetterling, Numerical Recipes: The Art of Scientific Computing, 2nd ed. (Cambridge University Press, Cambridge, England, 1992).

${ }^{39}$ C. J. Trahan, K. Hughes, and R. E. Wyatt, J. Chem. Phys. 118, 9911 (2003).

${ }^{40}$ E. R. Bittner, J. Chem. Phys. 119, 1358 (2003).

${ }^{41}$ M. F. Herman and E. Kluk, Chem. Phys. 91, 27 (1984).

${ }^{42}$ E. J. Heller, J. Chem. Phys. 75, 2923 (1981). 